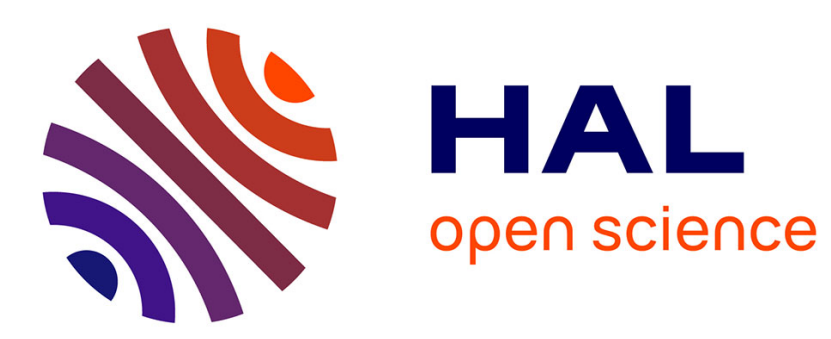

\title{
Automatic Optimal Biped Walking as a Mixed-Integer Quadratic Program
}

\author{
Aurélien Ibanez, Philippe Bidaud, Vincent Padois
}

\section{To cite this version:}

Aurélien Ibanez, Philippe Bidaud, Vincent Padois. Automatic Optimal Biped Walking as a MixedInteger Quadratic Program. Jadran Lenarčič, Oussama Khatib. Advances in Robot Kinematics, Springer International Publishing, pp.505-516, 2014, 978-3-319-06698-1. 10.1007/978-3-319-066981_52. hal-01030802

\section{HAL Id: hal-01030802 https://hal.science/hal-01030802}

Submitted on 22 Jul 2014

HAL is a multi-disciplinary open access archive for the deposit and dissemination of scientific research documents, whether they are published or not. The documents may come from teaching and research institutions in France or abroad, or from public or private research centers.
L'archive ouverte pluridisciplinaire HAL, est destinée au dépôt et à la diffusion de documents scientifiques de niveau recherche, publiés ou non, émanant des établissements d'enseignement et de recherche français ou étrangers, des laboratoires publics ou privés. 


\title{
Automatic optimal biped walking as a Mixed-Integer Quadratic Program
}

\author{
Aurelien Ibanez, Philippe Bidaud, and Vincent Padois
}

\begin{abstract}
This paper proposes an original Model Predictive Control approach to the walking control for humanoid robots, which allows to generate stable walking motions without the prior definition of footsteps positions and instants. Both the instant and amplitude of the changes in the supporting surface are part of the walking motion generation problem, and are described by a set of highly-constrained integer and real variables. Combined with the center of mass trajectory of the robot, this description leads to the formulation of a Mixed-Integer Quadratic Program in a Model predictive framework aiming at reaching high-level objectives, such as velocity tracking and tip-over risk minimization. The contribution of this approach is illustrated by the simulation of two scenarii, demonstrating the validity of the steps and trajectories computed in push-recovery and walking velocity tracking cases.
\end{abstract}

Key words: Biped walking, balance control, hybrid systems, footsteps planning, push recovery, Mixed-Integer Quadratic Programming.

\section{Introduction}

The works introduced in this paper address the control problem of safe biped walking, aiming at the predictive generation of stable motions without the use of prior gait strategy, such as footsteps positions or triggers, in response to high-level objectives and large disturbances.

Aurelien Ibanez $\cdot$ Philippe Bidaud · Vincent Padois

Sorbonne Universités, UPMC Univ Paris 06, UMR 7222, Institut des Systèmes Intelligents et de Robotique, F-75005, Paris, France

CNRS, UMR 7222, Institut des Systèmes Intelligents et de Robotique, F-75005, Paris, France

e-mail: \{ibanez,bidaud,padois\}@isir.upmc.fr

Philippe Bidaud is also with ONERA, 91123 Palaiseau, France 
Maintaining postural balance under a large physical disturbance may go through several types of strategies, ranging from body torquing and motion of the center of mass (CoM) to the adaptation of the base of support. It is clear that changes in the base of support, either by stepping or grasping an object, have the potential to provide a greater degree of stabilization than solely using inertial forces in a fixed-support configuration [4]. These changes might be necessary when the system undergoes strong physical disturbances or when required by the activity. If so, the best feet placement is a question which can find answers in the capture of the system's dynamics and kinematics with reduced models, as found in approaches such as Capture Region [5] or using Model Predictive Control [2]. Although the Capture Region method provides means to determine which strategy to adopt and when, the end of step and ongoing physical activity could potentially affect the system's ability to execute changes of support. A predictive approach may allow to capture such effects and act accordingly.

This paper proposes to describe the contact state of the system using a highly constrained, mixed-integer set of variables. The resulting MPC formulation of the problem takes the form of a computationnally efficient Mixed-Integer Quadratic Program (MIQP), which allows to preview and compute optimal changes of the supporting surface (i.e. feet positions over time) and CoM trajectory. Contribution of this approach is that walking motions are generated as a whole as the result of a quadratic problem, without the use of prior low-level inputs such as footsteps positions and instants, and relatively to an ongoing walking activity. This activity is simply specified as two high-level objectives, being balance and CoM tracking.

The method is introduced as follows. First, an original description of the contact state of a humanoid is introduced in order to bring a linear biped model subject to constraints related to the walking motion. This model is then involved in a MPC framework, leading to the definition of a MIQP. Simple results are finally presented to demonstrate the validity and contribution of this approach, in push-recovery and walking cases.

\section{Linear Mixed-Integer biped model}

The general approach of the method presented in this paper consists in finding admissible changes in the supporting surface, in order to ensure safe balance while tracking a desired behavior of CoM of the system. The balance state of the robot in walking motion can be captured by relating its CoM dynamics to its contact state. This section therefore aims at describing the robot state with a set of variables representing both its center of mass behavior and contact state. 


\subsection{Preliminaries: Mixed-Integer contact state description}

In walking motions without sliding, changes in contact state are discrete and thus subject to several constraints, as illustrated in Figure 1. The set of variables capturing the contact state of the robot should therefore be chosen adequately, in order to be able to express these constraints in a desirable form with a view to future optimization; linear constraints are generally preferred as feasibility is easily maintained in such problems.

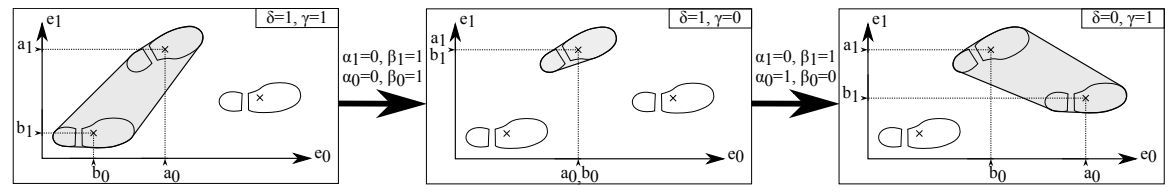

Fig. 1 Overview of the real-valued variables $a$ and $b$, along with the binary variables $\delta$ and $\gamma: a$ and $b$ bound the position of the feet in contact, $\gamma$ differentiates single and double support phases, and $\delta$ restrains the evolution of bounds $a, b$ during transitions from double to single support.

This section proposes a choice of linearly-constrained descriptors of the contact state. The supporting surface is characterized by its bounding box: this box is described, in the horizontal plane of the ground, by real-valued variables $(\boldsymbol{a}, \boldsymbol{b}) \in$ $\mathbb{R}^{2} \times \mathbb{R}^{2}$ defined as the upper and lower bounds, respectively, of the position in the two horizontal directions of centers of the feet in contact $^{1}$, as depicted in Figure 1.

Changes of these variables are subject to several constraints, called shape constraints, detailed in Appendix A. Shape constraints mainly enforce that variables $(\boldsymbol{a}, \boldsymbol{b})$ cannot change continuously: binary variables $(\boldsymbol{\alpha}, \boldsymbol{\beta}) \in\{0,1\}^{2} \times\{0,1\}^{2}$ are introduced as rising and falling edges of $\boldsymbol{a}$ and $\boldsymbol{b}$ respectively, in order to define constraints restraining the evolution of $(\boldsymbol{a}, \boldsymbol{b})$ to discrete changes solely.

Because variables $(\boldsymbol{a}, \boldsymbol{b})$ are implicitly related to feet positions, additional constraints, called admissibility constraints, must be brought. As illustrated in Figure 1 and detailed in Appendix B, binary variables $(\gamma, \delta) \in\{0,1\} \times\{0,1\}$ are introduced to express such constraints, differentiating single support phases from double support ones and relating feet configurations to bounds $(\boldsymbol{a}, \boldsymbol{b})$.

The resulting set $(\boldsymbol{a}, \boldsymbol{b}, \boldsymbol{\alpha}, \boldsymbol{\beta}, \boldsymbol{\delta}, \boldsymbol{\gamma})$ is therefore a linearly-constrained, redundant description of the contact state of the robot. The following section presents a complete biped model relating contact state to CoM dynamics in order to capture the balance state of the system.

In the rest of this paper, time is sampled at discrete control instants $t_{i}$, and notation $v_{j}$ for function $v$ of time $t$ denotes the value $v\left(t_{j}\right)$, and $v_{k \mid j}$ the value $v\left(t_{j}\right)$ estimated from control time $t_{k}$.

${ }^{1}$ This description implies that the feet contact and lift-off the ground parallel to it, and are rigid bodies. 


\subsection{Linear biped model}

The state $\xi$ of the robot in walking motion is described through its contact state $(\boldsymbol{a}, \boldsymbol{b}, \boldsymbol{\alpha}, \boldsymbol{\beta}, \boldsymbol{\delta}, \boldsymbol{\gamma})$ and CoM horizontal dynamics $(\boldsymbol{c}, \dot{\boldsymbol{c}}, \ddot{\boldsymbol{c}})$, where $\boldsymbol{c} \in \mathbb{R}^{3}$ is the position of the CoM in the world frame.

The major contribution of the description $(\boldsymbol{a}, \boldsymbol{b}, \boldsymbol{\alpha}, \boldsymbol{\beta}, \boldsymbol{\delta}, \boldsymbol{\gamma})$ of the contact state of the robot, introduced in Section 2.1, is that it can be shown that shape and admissibility constraints (15)-(24), acting on these state variables, can be put in linear form (equalities and inequalities) with respect to $\xi$ in discrete time. ${ }^{2}$ These constraints (15)-(24) can be written in the form

$$
\forall k \in \mathbb{N}, \quad \mathbf{A}_{c, l} \boldsymbol{\xi}_{k}+\mathbf{A}_{c, r} \boldsymbol{\xi}_{k+1} \preceq \boldsymbol{f}_{c},
$$

where $\mathbf{A}_{c, l}$ and $\mathbf{A}_{c, r}$ are $n_{c} \times 19$ matrices, and $\boldsymbol{f}_{c}$ a vector in $\mathbb{R}^{n_{c}}$.

However, validity of the contact state with respect to the system dynamics requires that the center of pressure lies within the convex hull of the supporting surface. In these works, the position of the CoP on the ground is approximated as in the ZMP Preview Control framework [3], i.e. neglecting rotational effects and thus only considering inertial effects resulting from the dynamics of the center of mass of the system. ${ }^{3}$ The position $\boldsymbol{p} \in \mathbb{R}^{2}$ of the CoP on the ground can hence be derived from the CoM dynamics as, with $g$ the gravity acceleration, $\boldsymbol{p}=\boldsymbol{h}-\frac{\boldsymbol{c} \cdot \boldsymbol{e}_{2}}{g} \ddot{\boldsymbol{h}}$, where $\boldsymbol{e}_{2}$ is the ascendant vertical direction and $h=c-\left(c \cdot e_{2}\right) e_{2}$ the horizontal position of the CoM. We recall that validity of the model imposes that $\boldsymbol{p}$ lies within the convex hull of the supporting surface. Although this inclusion constraint is linear with respect to the horizontal dynamics of the CoM, the fact that changes in the supporting surface are reflected by changes in state variables $(\boldsymbol{a}, \boldsymbol{b})$ brings quadratic terms. Nevertheless, as shown in [2], overestimating the convex hull of this surface by its bounding box in forward and lateral directions brings the definition of a set of linear inequality constraints with respect to the state $\boldsymbol{\xi}$ of the system. Under this overestimation, the CoP constraints write

$$
\forall k \in \mathbb{N}, \quad \mathbf{A}_{p} \boldsymbol{\xi}_{k} \preceq \boldsymbol{f}_{p},
$$

where $\mathbf{A}_{p}$ is a $n_{p} \times 19$ matrix, and $\boldsymbol{f}_{p}$ a vector in $\mathbb{R}^{n_{p}}$.

The biped model finally writes at time $t_{k}$

$$
\begin{aligned}
& \forall k \in \mathbb{N}, \quad \boldsymbol{\xi}_{k}=\left[\begin{array}{llllllllll}
\boldsymbol{a}_{k} & \boldsymbol{b}_{k} & \boldsymbol{\alpha}_{k} & \boldsymbol{\beta}_{k} & \boldsymbol{\delta}_{k} & \gamma_{k} & \boldsymbol{c}_{k} & \dot{\boldsymbol{c}}_{k} & \ddot{\boldsymbol{c}}_{k}
\end{array}\right]^{T} \\
& \text { s.t. }\left\{\begin{array}{rlrl}
\mathbf{A}_{c, r} \boldsymbol{\xi}_{k} & \preceq \boldsymbol{f}_{c}-\mathbf{A}_{c, l} \boldsymbol{\xi}_{k-1}, & \mathbf{A}_{p} \boldsymbol{\xi}_{k} \preceq \boldsymbol{f}_{p}, \\
\left(\boldsymbol{\alpha}_{k}, \boldsymbol{\beta}_{k}\right) & \in\{0,1\}^{2} \times\{0,1\}^{2}, & \left(\boldsymbol{\delta}_{k}, \gamma_{k}\right) & \in\{0,1\} \times\{0,1\}, \\
\left(\boldsymbol{a}_{k}, \boldsymbol{b}_{k}\right) & \in \mathbb{R}^{2} \times \mathbb{R}^{2}, & \left(\boldsymbol{c}_{k}, \dot{\boldsymbol{c}}_{k}, \ddot{\boldsymbol{c}}_{k}\right) & \in \mathbb{R}^{3} \times \mathbb{R}^{3} \times \mathbb{R}^{3} .
\end{array}\right.
\end{aligned}
$$

\footnotetext{
${ }^{2}$ Under the hypothesis that there exists upper bounds for the difference $\|a-b\|_{1}$ and for the variations of $a$ and $b$ between two consecutive instants. This hypothesis is always verified in the case of a biped robot which is subject to geometrical constraints.

${ }^{3}$ Moreover, vertical dynamics of the center of mass are also neglected and this hypothesis needs to be enforced in the whole-body control of the robot.
} 


\subsection{High-level walking motion constraints}

A selection of additional high-level constraints, related to the walking motion in itself and limitations of the robot, are introduced in the problem.

Maximal leg span and step length can be easily put in the form of linear inequality constraints with respect to the system state $\boldsymbol{\xi}$. Additionally, minimum durations of single and double support phases can be enforced, in the form of a set of linear inequality constraints, in order to avoid the generation of small successive steps. Last, the ratio between step size and single support duration can be bounded by a maximal swinging foot velocity implemented as a set of linear inequality constraints, constraints that will hinder solutions requiring an excessively fast movement of the swinging foot in the transition between two distinct double support phases.

Let $\bar{v}$ denote the maximal swinging foot velocity, $\bar{s}$ the maximal step length and $\bar{t}$ the largest of minimum durations of single and double support phases, these high-level constraints write

$$
\forall k \in \mathbb{N} \quad \sum_{t_{k+j}-t_{k} \leq \max (\bar{s} / \bar{v}, \bar{t})} \mathbf{A}_{w, j} \boldsymbol{\xi}_{k+j} \preceq \boldsymbol{f}_{w}, \quad j \in \mathbb{N},
$$

where $\mathbf{A}_{w, j}$ is a $n_{w} \times 19$ matrix, and $\boldsymbol{f}_{w}$ a vector in $\mathbb{R}^{n_{w}}$ depending on a history of states $\boldsymbol{\xi}$ prior to $t_{k}$. It can be shown that $\boldsymbol{f}_{w}$ can be written in the form

$$
\boldsymbol{f}_{w}=\boldsymbol{f}_{w_{0}}+\sum_{t_{k}-t_{k-j}<\max (\bar{s} / \bar{v}, \bar{t})} \mathbf{B}_{w, j} \boldsymbol{\xi}_{k-j}, \quad j \in \mathbb{N}^{*}
$$

\section{Quadratic Mixed-Integer Predictive Control}

The control problem of walking motion generation is formulated as follows: find, over a preview horizon, admissible changes in the support surface (foot steps position and instants) and optimal center of mass trajectory (balance adjustments) that minimize, among other secondary objectives, a tracking error with a desired center of mass path and velocity (stand still, walk forward, etc.) and maximize the distance of the CoP to the bounds of the supporting surface (minimum tip-over risk).

\subsection{Quadratic Model Predictive Control}

The aim of the balance MPC problem is to find at each control step $t_{k}$, in addition to admissible changes in the supporting surface, a horizon of CoM trajectory that minimizes both a tracking error to a reference trajectory (desired displacements of the system as a whole) and a tip-over risk. Due to the hypotheses of the ZMP-based balance criterion, a horizontal trajectory solely is of interest in such a problem.

A future trajectory $\left(\boldsymbol{h}_{k \mid k+j}, \dot{\boldsymbol{h}}_{k \mid k+j}, \ddot{\boldsymbol{h}}_{k \mid k+j}\right)_{j=1 . . N}$ of the center of mass, previewed from state $\boldsymbol{\xi}_{k}$ at control step $t_{k}$ over $N$ time steps, can be entirely defined 
from a horizon of $N$ piecewise constant CoM jerks $\boldsymbol{u}=\partial^{3} \boldsymbol{h} / \partial t^{3}$, using a straightforward discrete integration scheme. With $\hat{\boldsymbol{h}}=[\boldsymbol{h} \dot{\boldsymbol{h}} \ddot{\boldsymbol{h}}]^{T}$, the CoM preview writes

$$
\forall(k, j) \in \mathbb{N} \times \mathbb{N}, \quad \hat{\boldsymbol{h}}_{k \mid k+j+1}=\mathbf{A}_{h} \hat{\boldsymbol{h}}_{k \mid k+j}+\mathbf{B}_{h} \boldsymbol{u}_{k \mid k+j+1},
$$

where $\mathbf{A}_{h}$ and $\mathbf{B}_{h}$ are integration matrices. Under the assumption that the altitude of the CoM remains constant over time, a previewed state $\boldsymbol{\xi}_{k \mid k+j+1}$ can be linearly derived from previous state $\boldsymbol{\xi}_{k \mid k+j}$ given the value $\boldsymbol{\chi}_{k \mid k+j+1}$ of the unknown variable defined as

$$
\chi=\left[\begin{array}{llllllll}
\boldsymbol{a} & \boldsymbol{b} & \boldsymbol{\alpha} & \boldsymbol{\beta} & \delta & \gamma & \boldsymbol{u}
\end{array}\right]^{T} .
$$

Notation (7) and relation (6) indeed brings, with $\mathbf{Q}$ and $\mathbf{T}$ state description matrices, the linear time-invariant process

$$
\forall(k, j) \in \mathbb{N} \times \mathbb{N}, \quad \boldsymbol{\xi}_{k \mid k+j+1}=\mathbf{Q} \boldsymbol{\xi}_{k \mid k+j}+\mathbf{T} \boldsymbol{\chi}_{k \mid k+j+1},
$$

which allows the preview, from the actual state $\boldsymbol{\xi}_{k \mid k}=\boldsymbol{\xi}_{k}$, of a horizon $\boldsymbol{C}_{k, N}$ of $N$ future states $\boldsymbol{\xi}_{k \mid k+j}$ from a horizon $\boldsymbol{X}_{k, N}$ of $N$ future inputs $\chi_{k \mid k+j}$. Indeed, Equation (8) can be spread over $N$ steps and bring

$$
\boldsymbol{C}_{k, N}=\left[\begin{array}{c}
\boldsymbol{\xi}_{k \mid k+1} \\
\vdots \\
\boldsymbol{\xi}_{k \mid k+N}
\end{array}\right]=\mathbf{P} \boldsymbol{\xi}_{k}+\mathbf{R}\left[\begin{array}{c}
\chi_{k \mid k+1} \\
\vdots \\
\chi_{k \mid k+N}
\end{array}\right]=\mathbf{P} \boldsymbol{\xi}_{k}+\mathbf{R} \boldsymbol{X}_{k, N},
$$

and thus constraints (1), (2) and (4) can be rewritten

$$
\mathbf{A} \boldsymbol{X}_{k, N} \preceq \boldsymbol{f}
$$

where $f$ depends on a history of states $\boldsymbol{\xi}$, as introduced in (1), (4) and (5).

The minimization of tip-over risk can be expressed at the level of the center of pressure: safe balance motor control should aim at maximizing the distance between the $\mathrm{CoP}$ and the convex hull of the supporting surface. ${ }^{4}$ This objective can be expressed as the cost function $J_{b}$ to be minimized

$$
J_{b k}=\sum_{j=1}^{N}\left\|p_{k \mid k+j}-r_{k \mid k+j}\right\|^{2}
$$

which measures a cumulated square distance between the CoP and the center $r$ of the supporting surface, where $\boldsymbol{r}=(\boldsymbol{a}+\boldsymbol{b}) / 2$.

Walking motions generally aim at reaching a desired horizontal displacement of the system as a whole (null displacement in the case of standstill activities), that can be expressed as a reference CoM horizontal trajectory $\hat{\boldsymbol{h}}^{\text {ref }}$. This second objective can be reached through the minimization of the cost function $J_{w}$ that writes

$$
J_{w k}=\sum_{j=1}^{N}\left\|\mathbf{S}\left(\hat{\boldsymbol{h}}_{k \mid k+j}-\hat{\boldsymbol{h}}_{k \mid k+j}^{r e f}\right)\right\|^{2},
$$

where $\mathbf{S}$ is a $6 \times 6$ weighting selection matrix, diagonal, defining whether position, velocity and/or acceleration are tracked in each of the two horizontal directions.

Secondary objectives can be added to the problem in the form of the quadratic $\operatorname{cost} q$, for regulation purposes or additional input, for example in order to prefer double

${ }^{4}$ As a tip-over case (instantaneous rotation around a point on the ground) is the result of the CoP being on the edge of the convex hull of the supporting surface. 
support phases over single support ones.

Objectives (11) and (12) are quadratic with respect to $\boldsymbol{X}_{k, N}$, and can be synthesized in the form

$$
J_{k}=\omega_{b} J_{b k}+\omega_{w} J_{w k}+q=\boldsymbol{X}_{k, N}^{T} \mathbf{H} \boldsymbol{X}_{k, N}+\boldsymbol{d}^{T} \boldsymbol{X}_{k, N}
$$

where $\mathbf{H}$ is a positive definite matrix, $\boldsymbol{d}$ a vector and $\left(\omega_{b}, \omega_{w}\right)$ scalar weights. The MPC problem finally writes

$$
\begin{aligned}
& \min _{\boldsymbol{X}_{k, N}} \boldsymbol{X}_{k, N}^{T} \mathbf{H} \boldsymbol{X}_{k, N}+\boldsymbol{d}^{T} \boldsymbol{X}_{k, N} \\
& \text { s.t. }\left\{\begin{array}{rlrl}
\mathbf{A} \boldsymbol{X}_{k, N} & \preceq \boldsymbol{f} \\
\boldsymbol{\xi}_{k \mid k}=\boldsymbol{\xi}_{k}, \quad \boldsymbol{\xi}_{k \mid k+j} & =\mathbf{Q} \boldsymbol{\xi}_{k \mid k+j-1}+\mathbf{T} \boldsymbol{\chi}_{k \mid k+j}, j=1 . . N \\
\left(\boldsymbol{a}_{k \mid k+j}, \boldsymbol{b}_{k \mid k+j}, \boldsymbol{u}_{k \mid k+j}\right) & \in \mathbb{R}^{2} \times \mathbb{R}^{2} \times \mathbb{R}^{2}, & j=1 . . N \\
\left(\boldsymbol{\alpha}_{k \mid k+j}, \boldsymbol{\beta}_{k \mid k+j}\right) & \in\{0,1\}^{2} \times\{0,1\}^{2}, & j=1 . . N \\
\left(\boldsymbol{\delta}_{k \mid k+j}, \gamma_{k \mid k+j}\right) & \in\{0,1\} \times\{0,1\}, & & j=1 . . N
\end{array}\right.
\end{aligned}
$$

which is a Mixed-Integer Quadratic Program. Note that the complexity of problem (14), relatively to a standard QP, is strongly related to the number of integer variables. Binary variables $(\boldsymbol{\alpha}, \boldsymbol{\beta}, \boldsymbol{\delta}, \boldsymbol{\gamma})$ can be discretized with a larger time increment than real-valued variables, and the previous formulation still holds given appropriate re-sampling matrices, while reducing the size of problem (14). The only drawbacks of such an approach is that it restrains, in the preview solely, the occurrence of changes in the supporting surface to fewer potential instants.

\section{Results}

The validity of the mixed-integer predictive formulation (14) of the walking control problem is demonstrated through the simulation of two common biped scenarii: push recovery and forward walking. In both cases, motion of the center of mass and of the supporting contacts are automatically computed at each control step, as an optimal response to high-level CoM velocity tracking and balance objectives $J_{w}$ and $J_{b}$. In both scenarii, weights $\omega_{w}$ and $\omega_{b}$ are equal.

Simulations are performed using the ARBORIS-PYTHON simulator [1] developed at ISIR, and whole-body motion from optimal outputs of the MPC (14) is ensured by an LQP-based controller [6] for an iCub [7] robot model. The system state is previewed over a horizon of $1.0 \mathrm{~s}$.

The push-recovery scenario is specified as follows: the system must track a null CoM velocity $\left\|\dot{\boldsymbol{h}}^{r e f}\right\|=0 m \cdot s^{-1}$ while maintaining balance, and an unknown force $\boldsymbol{F}^{\text {waist }}=[60 N-20 N]$ is applied to the waist of the robot during a period of $0.1 \mathrm{~s}$. Outputs from (14) automatically compensate the sudden changes in the system state resulting from this external force by triggering a sequence of footsteps previewed over a future horizon. As the system reaches its desired state $\|\dot{\boldsymbol{h}}\|=0 m \cdot \mathrm{s}^{-1}$, the optimal solution of (14) requires no further steps. An overview of the results is presented on Figure 2. 

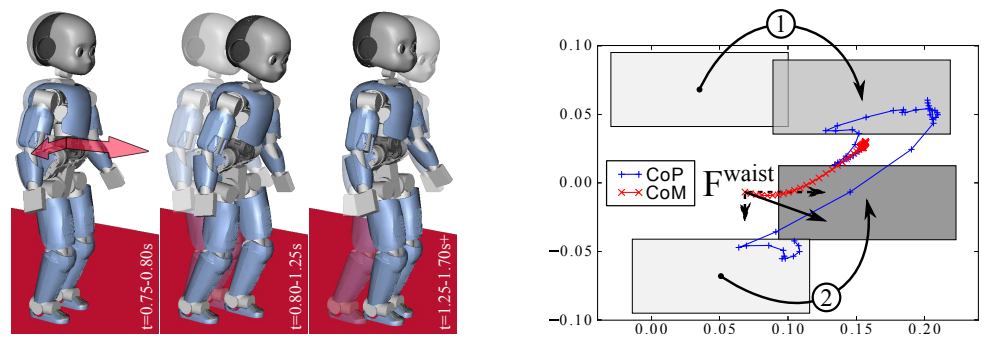

Fig. 2 Overview of the results from the MIQP predictive framework (14) - left: optimal automatic push-recovery steps - right: evolution of the CoP and CoM of the robot after impact $F^{\text {waist }}$.
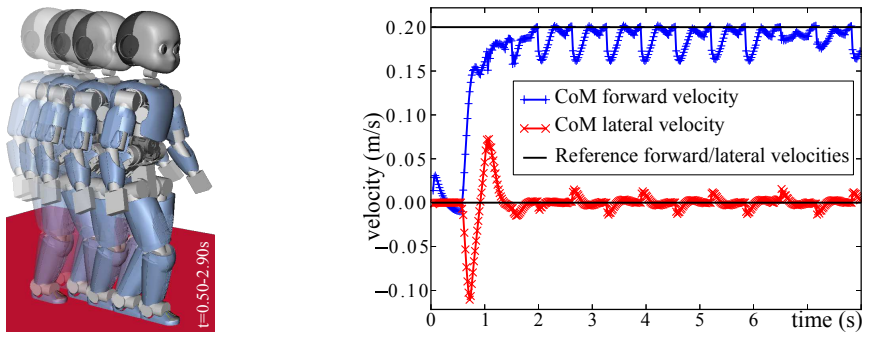

Fig. 3 Overview of the results from the MIQP predictive framework (14) - left: optimal automatic gait from high-level velocity tracking - right: minimization of the CoM velocity tracking objective.

In the walking scenario, a velocity $\dot{\boldsymbol{h}}^{\text {ref }}=\left[0.20 \mathrm{~m} \cdot \mathrm{s}^{-1} 0 \mathrm{~m} \cdot \mathrm{s}^{-1}\right]$ is set as a reference. This reference states that the system must move forward while minimizing lateral velocity of its CoM. Results shown on Figure 3 show that the generated gait effectively tracks the desired velocity in both lateral and forward directions. An interesting result is that optimal footsteps of such a problem are aligned in order to minimize velocity of the CoM in the lateral direction. Note that collision avoidance constraints and objectives might challenge this result.

\section{Conclusion}

The Model Predictive Control framework introduced in this paper allows the generation of optimal walking motions for biped robots, able to reach high-level objectives such as velocity tracking and tip-over risk minimization, without the use of prior heuristics such as predefined feet positions or footsteps triggers.

The contact state of the robot is described through a mixed set of variables involving integers, conjointly optimized with the CoM horizontal trajectory over a preview horizon in order to maintain balance while tracking high-level objectives.

The validity of the resulting MIQP formulation is demonstrated in push-recovery and walking speed tracking scenarii, exhibiting automatic footsteps computation and trigger. 
Future works will investigate different behaviors in more complex cases, while providing more insights on the computational cost of such problems.

\section{Appendix A}

Variables $\boldsymbol{a}$ and $\boldsymbol{b}$ are piecewise constant functions of time $\mathrm{s}^{5}$; binary variables $(\boldsymbol{\alpha}, \boldsymbol{\beta}) \in\{0,1\}^{2} \times\{0,1\}^{2}$ are therefore introduced as rising and falling edges of $\boldsymbol{a}$ and $\boldsymbol{b}$ respectively, defined as follows

$$
\forall i \in\{0,1\}, \forall t \in \mathbb{R}, \quad\left\{\begin{array}{lll}
\alpha_{i}(t)=1 & \Rightarrow & \alpha_{i}\left(t^{-}\right)=\alpha_{i}\left(t^{+}\right)=0 \\
\alpha_{i}(t)=0 & \Rightarrow & a_{i}\left(t^{-}\right)=a_{i}\left(t^{+}\right)=a_{i}(t)
\end{array}\right.
$$

and similarly for the pair $(\boldsymbol{\beta}, \boldsymbol{b})$. Variables $\boldsymbol{\alpha}, \boldsymbol{\beta}$ can have their value set to 1 at distinct instants solely (15), and $\boldsymbol{\alpha}=0$ imposes constancy of $\boldsymbol{a}$ as written in (16). Note that changes in $\boldsymbol{a}$ and $\boldsymbol{b}$ are subject to the following additional constraints

$$
\begin{aligned}
\forall i \in\{0,1\}, \forall t \in \mathbb{R}, & b_{i}(t) \leq a_{i}(t), \\
\forall i \in\{0,1\}, \forall t \in \mathbb{R}, & \alpha_{i}(t)+\beta_{i}(t) \leq 1, \\
\forall t \in \mathbb{R}, & \alpha_{0}(t)+\beta_{0}(t)=\alpha_{1}(t)+\beta_{1}(t) .
\end{aligned}
$$

The definition of $\boldsymbol{a}$ and $\boldsymbol{b}$ as respectively upper and lower bounds has to be enforced (17); also, as only one foot can move at a time, it imposes that, in a given direction, only one of the bounds $\boldsymbol{a}$ or $\boldsymbol{b}$ can change (18). Last, a change in a first direction for either bound $\boldsymbol{a}$ or $\boldsymbol{b}$ must allow a change in the other direction (19).

Constraints (15)-(18) define the bounding box of the support surface as restricted between two bi-dimensional, piecewise constant variables $\boldsymbol{a}$ and $\boldsymbol{b}$. However, the nature of the changes occurring in $\boldsymbol{a}$ and $\boldsymbol{b}$ imposes the consideration of additional variables and constraints to define and guarantee the admissibility of such changes.

\section{Appendix B}

As shown in Figure 1, a phase of single support requires that $\boldsymbol{a}$ and $\boldsymbol{b}$ are equal, and phases of double support (DS) are necessarily separated by, at least, a single support phase (SS). Binary variable $\gamma \in\{0,1\}$ is therefore introduced to capture the alternation between single and double support phase: $\gamma=1$ describes a double support phase and $\gamma=0$ a single support one. The single support constraint writes

$$
\forall t \in \mathbb{R}, \gamma(t)=0 \quad \Rightarrow \quad \boldsymbol{a}(t)=\boldsymbol{b}(t),
$$

and the alternation ones, accounting for (19)

$$
\alpha_{0}(t)+\beta_{0}(t)=1 \Rightarrow \gamma(t)=\left\{\begin{array}{lll}
0 & \text { if } & \gamma\left(t^{-}\right)=1 \\
1 & \text { if } & \gamma\left(t^{-}\right)=0
\end{array}\right.
$$

\footnotetext{
${ }^{5}$ The discrete nature of contact states in non-sliding walking motions imposes that changes in $\boldsymbol{a}$ and $\boldsymbol{b}$ can only occur at discrete events.
} 
and

$$
\alpha_{0}(t)+\beta_{0}(t)=0 \Rightarrow \gamma(t)=\gamma\left(t^{-}\right),
$$

which state that $\gamma$ must change if and only if a change in variables $\boldsymbol{a}$ or $\boldsymbol{b}$ occurs. ${ }^{6}$ However, these variables and constraints are not sufficient to guarantee the admissibility of changes in the bounding box described by $(\boldsymbol{a}, \boldsymbol{b})$. Indeed, as shown in Figure 1, potential changes in $\boldsymbol{a}$ and $\boldsymbol{b}$ from a DS phase to a SS one depend on the configuration of the feet, relatively to bounds $\boldsymbol{a}$ and $\boldsymbol{b}$, in the DS phase. If one of the two feet in contact defines the upper bound $\boldsymbol{a}$ in both directions ( $c f$. Figure 1, far left), the following single support phase can only result from a change of either $\boldsymbol{a}$ or $\boldsymbol{b}$ in both directions ; symmetrically, if one of the two feet in contact defines the upper bound $\boldsymbol{a}$ in one direction and lower bound $\boldsymbol{b}$ in the other ( $c f$. Figure 1, far right), the following SS phase will result from a change in $\boldsymbol{a}$ in one direction, and $\boldsymbol{b}$ in the other. This time history constraint can be captured by the introduction of the binary variable $\delta \in\{0,1\}$, having 1 for value in the first case and 0 in the second.The indicator of configuration $\delta$ must remain constant through each DS phase in order to keep the information for the next transition towards a SS phase; that is

$$
\forall t \in \mathbb{R}, \gamma(t)=1 \quad \Rightarrow \quad \delta\left(t^{+}\right)=\delta(t),
$$

and, as described earlier, changes in $a$ and $b$ are related to the values of $\delta$. This relation writes

$$
\forall t \in \mathbb{R}, \delta(t)=1 \Rightarrow\left\{\begin{array}{l}
\alpha_{0}(t)=\alpha_{1}(t) \\
\beta_{0}(t)=\beta_{1}(t)
\end{array} \text {, and } \delta(t)=0 \Rightarrow\left\{\begin{array}{l}
\alpha_{0}(t)=\beta_{1}(t) \\
\beta_{0}(t)=\alpha_{1}(t)
\end{array}\right.\right.
$$

Note that this relation is bilateral : if $\delta$ is unconstrained by (23), changes in $\boldsymbol{a}$ and $\boldsymbol{b}$ define the value of $\delta$ and else, the value of $\delta$ restrains potential changes in $\boldsymbol{a}$ and $\boldsymbol{b}$.

\section{References}

1. Barthelemy, S., Salini, J., Micaelli, A.: Arboris-python. URL https://github.com/salini/arborispython

2. Herdt, A., Diedam, H., Wieber, P.B., Dimitrov, D., Mombaur, K., Diehl, M.: Online walking motion generation with automatic footstep placement. In: Adv. Rob., vol. 24, pp. 719-737 (2010)

3. Kajita, S., Kanehiro, F., Kaneko, K., Kajiwara, K., Harada, K., Yokoi, K., Hirukawa, H.: Biped walking pattern generation by using preview control of zero-moment point. In: Proc. of the IEEE ICRA (2003)

4. Maki, B.E., Mcilroy, W.E., Fernie, G.R.: Change-in-support reactions for balance recovery. Engineering in Medicine and Biology Magazine, IEEE 22(2), 20-26 (2003)

5. Pratt, J., Carff, J., Drakunov, S., Goswami, A.: Capture point: A step toward humanoid push recovery. In: Proc. of the IEEE-RAS Int. Conf. on Humanoid Rob., pp. 200-207. IEEE (2006)

6. Salini, J., Padois, V., Bidaud, P.: Synthesis of complex humanoid whole-body behavior: a focus on sequencing and tasks transitions. In: Proc. of the IEEE ICRA, pp. 1283-1290. IEEE (2011)

7. Sandini, G., Metta, G., Vernon, D.: The icub cognitive humanoid robot: An open-system research platform for enactive cognition. In: 50 Years of Artificial Intelligence, Lecture Notes in Computer Science, chap. 32, pp. 358-369. Springer (2007)

\footnotetext{
${ }^{6}$ Note that variable $\gamma$ is thus fully defined through variables $\boldsymbol{\alpha}$ and $\boldsymbol{\beta}$, given some initial conditions.
} 